\title{
Identification of Spatiotemporal Dispersion Electrograms in Atrial Fibrillation Ablation Using Machine Learning: A comparative Study
}

\author{
Amina Ghrissi ${ }^{1}$, Douglas Almonfrey ${ }^{2}$, Fabien Squara ${ }^{1,3}$, Johan Montagnat ${ }^{1}$ and Vicente Zarzoso ${ }^{1}$
}

\begin{abstract}
Atrial Fibrillation (AF) is the most widespread sustained arrhythmia in clinical practice. A recent personalized AF therapy consists in ablating areas displaying spatiotemporal dispersion (STD) electrograms (EGM) with the use of catheters. Interventional cardiologists use a multipolar mapping catheter called PentaRay to identify visually atrial sites with STD pattern by visual inspection. In this contribution, we propose to automatize the identification of STD EGMs using machine learning while comparing several features. The aim is to design a data representation and an adapted classification algorithm for accurate EGM detection with affordable computational resources and low prediction time. Four data formats are considered: 1) EGM matrices; 2) EGM plots; 3) three-dimensional EGM plots; 4) maximal voltage absolute values. Convolutional neural networks and transfer learning based on the VGG16 architecture are benchmarked. Classification results on the test set show that extracting features automatically with VGG16 is possible and yields comparable results to classifying raw EGM recordings with values of accuracy and AUC of $90 \%$. However, the overall precision and $F 1$ score are low $(50 \%)$, which can be explained by the high class imbalance ratio. This issue is addressed with data augmentation. Due to its low computational cost, our solution can also be deployed in real time.

Index Terms-Atrial fibrillation, spatiotemporal dispersion, catheter ablation, multipole catheter, multichannel electrogram, machine learning, transfer learning.
\end{abstract}

\section{INTRODUCTION}

During the last decade, artificial intelligence models [1], [2] have intensively been used as decision-aid solutions in biomedical data analysis. In the current study, we are interested in helping cardiologists to automatically identify potential ablation sites in persistent atrial fibrillation (AF) using machine learning (ML) tools. AF represents the most frequent sustained arrhythmia experienced in clinical practice and it rises in prevalence with advancing age [3]. AF is associated with a 5-fold stroke risk increase and a 2-fold increased risk of both mortality and dementia [4]. Hence, AF entails an important economic burden. Among the existing treatments of persistent $\mathrm{AF}$, ablation interventions beat drug therapies in terms of long-term effectiveness. Ablation consists in burning with radiofrequency (RF) energy cardiac tissue areas estimated to be responsible for the presence

This work was supported in part by the French government Investments in the Future program, through IDEX UCA ${ }^{\text {JEDI }}$ (ANR-15-IDEX-0001) and 3IA Côte d'Azur (ANR-19-P3IA-0002) projects. V. Zarzoso holds the Chair "IAblation" from 3IA Côte d'Azur.

1 Université Côte d'Azur, CNRS, I3S Laboratory, Sophia Antipolis, France (e-mail: \{amina.ghrissi, vicente.zarzoso, fabien.squara, johan.montagnat\}@univ-cotedazur.fr)

2 Federal Institute of Espírito Santo, Vitoria, Brazil (e-mail: dalmonfreyeifes.edu.br).

3 Université Côte d'Azur, CHU Pasteur, Cardiology Department, Nice, France (e-mail: squara.f@chu-nice.fr). and maintenance of AF. The classical ablation approach, called sequential stepwise ablation, yields poor clinical results [5]. A novel wholly patient-tailored ablation protocol, giving $95 \%$ of procedural success rate, consists in identifying ablation sites based on a signal pattern called spatiotemporal dispersion (STD) [6]. Multipolar mapping catheters are used to record electrograms (EGM) in the atria thus targeting areas of STD as potential AF drivers. The high-density PentaRay catheter is used for STD localization. It has a five-branch star design with two bipoles on each spline. According to preliminary guidelines for STD identification from visual inspection, the 10-channel EGM recording acquired by the PentaRay would display a cardiac activation delay of $70 \%$ of AF cycle length (AFCL) on a minimum of three neighboring bipoles (channels) [6]. However, this visual inspection may be biased by the difficulty for the interventional cardiologist to precisely quantify the STD pattern at each single mapped location in real time, as hundreds and even thousands of cardiac sites are mapped in a typical ablation intervention.

To overcome these limitations, the present contribution aims to design a decision-aid solution that helps interventional cardiologists detect STD patterns automatically thanks to both baseline ML and modern deep learning tools [7]. We seek the most optimal EGM classification model, formed by the combination of a data representation and an adapted classification algorithm, in order to detect STD locations with the highest performance and lowest computational cost in terms of prediction time and resources. The study dataset includes a cohort of over 35000 10-channel EGM signals acquired from 15 different persistent AF patients.

Our preliminary results in a previous study on automatic detection of STD from multichannel EGM recordings were promising [8]. The classification performance on the test dataset reached $90 \%$ of accuracy and $80 \%$ of AUC using a shallow convolutional neural network $(\mathrm{CNN})$. But values of precision were low because of the lack of STD samples. We also addressed the issue of class imbalance and lack of training samples from STD class through adapted data augmentation (DA) methods [9] [10]. In a complementary recent study, we addressed the classification of handcrafted features from EGM data [11]. As suggested in [6], we studied a time series computed from multichannel EGM signals to perform STD detection. The time series is called maximal voltage PentaRay absolute values (VAVp). Classification results were promising in simulated data but disappointing in real data.

The present contribution updates, completes and consolidates our preliminary results in [8], [11] by putting 
forward a feature selection technique for a more optimal EGM classification solution. For this purpose, we design and benchmark different data representations: 1) raw EGMs stored in matrices; 2) two-dimensional (2D) images obtained by subplotting the curves of the 10 leads one under the other, as it is currently done by the mapping system in the hospital; 3 ) three-dimensional (3D) image, tensor as a result of stacking three shifted images of EGM curves; 4) one-dimensional (1D) VAVp time series used as a compact representation of the multichannel recordings. These data representations are combined with suitable classifiers including multivariate logistic regression (MLR) [2], dimensionality reduction with principal component analysis (PCA) [12] followed by support vector machines (SVM) [13], CNN [14], transfer learning (TL) [7] of the VGG16 model [15]. The originality of this contribution is three-fold: first, to our knowledge this is the first systematic application of state-of-the-art ML techniques to EGM classification for STD identification; second, different data representations are benchmarked and TL is applied for the first time to the identification of dispersion pattern in multichannel EGMs; third, our decisionaid solution can be implemented in real time with moderate computational resources, thus potentially improving catheter ablation success rates while reducing the duration of STDbased ablation interventions for treating persistent AF.

\section{Af Ablation}

\section{A. Catheter Ablation of Persistent AF}

1) AF Diagnosis: The heart is a vital muscle that pumps blood to irrigate the body thus providing oxygen and nutrients to body tissues. It is composed of four chambers, the two upper ones are called atria and the two lower ones are called ventricles. However, the heart mechanisms for a patient suffering from $\mathrm{AF}$ is different from that of a normal one, said to be in sinus rhythm. AF is characterized by an irregular activation in the atria that start quivering or fibrillating, causing non-synchronous fluctuations in the associated electrical baseline. Therefore, the ventricular rate becomes more rapid and disorganized [16].

2) $A F$ Therapies: The severity degrees of AF differ according to the arrhythmogenic episode duration and the response to treatment. In case of persistent AF, pharmacotherapy proves less effective than catheter ablation due to the complexity of this arrhythmia. Ablation is an invasive procedure that consists in burning the cardiac myocytes displaying irregularities with RF energy delivered with the use of catheters. The classical ablation protocol of persistent AF uses bipolar mapping catheters. It is called stepwise and consists: in 1) burning the triggers around the pulmonary veins thought to be responsible for initiating AF; 2) ablating areas of the atrial substrate harboring drivers maintaining and self-perpetuating the arrhythmia, such as complex fractionated electrograms (CFAE) [17]. However, a growing number of reports show the limitations of the stepwise approach, as several ablation interventions are typically required to terminate $\mathrm{AF}$ or at least to transform it to a more stable tachycardia [5].

\section{B. STD-Guided Ablation}

A recent discovery in AF ablation therapy shows that targeting only cardiac areas displaying STD EGMs can terminate AF in $95 \%$ of a cohort of 105 patients [6]. The resulting recurrence rate within 18 months of follow-up is only $15 \%$. STD is a visually discernible AF pattern that guides interventional cardiologists in ablating persistent AF drivers. STDbased ablation uses PentaRay mapping catheter (Biosense Webster Inc, Irvine, CA, USA) as shown in Fig. 1(A). Before ablation with RF energy, interventional cardiologists first position sequentially the PentaRay in different sites of the atria. Ten bipolar EGMs are then simultaneously recorded per location by maintaining the catheter stable for a few seconds. Finally, atrial sites displaying an irregular cardiac activity are annotated as dispersion points and tagged for ablation. According to guidelines in [6], dispersion areas refer to clusters of electrograms, either fractionated or not, displaying interelectrode time and space dispersion at a minimum of three contiguous leads [6], as shown in Fig. 1.(B). Hence, STD-based ablation is a fully patient-tailored therapy.

However, quantifying visually if the delay of the intracardiac activation through neighboring bipoles can be approximated by $70 \%$ of the AFCL is a difficult and timeconsuming task. Thousands of atrial locations are mapped with the PentaRay catheter in a typical ablation intervention and all multichannel EGM recordings acquired at these locations must be evaluated visually by the practicing cardiologists in real time. Hence, the visual identification of STD areas is prone to errors and lack of reproducibility. Instead, the present work aims to design an optimized decision-aid solution that helps interventional cardiologists detect STD patterns automatically thanks to ML tools.

\section{METHODS}

\section{A. Data Acquisition}

The process of data acquisition consists in several steps: 1) selecting patients suffering from persistent $\mathrm{AF}$ and mapped with PentaRay for STD-based ablation; 2) exporting intervention data from BioSense CARTO system, including electrocardiogram (ECG), EGM and annotated dispersion points; 3) decompressing, anonymizing and preparing data of interest; 4) structuring data for classification purposes.

During the mapping phase, the PentaRay catheter is maintained stable for $2.5 \mathrm{~s}$ at each location. A location refers to an anatomical point inside the heart. Multichannel EGMs are sampled at $1 \mathrm{kHz}$ and displayed through the CARTO system monitor, to be analyzed by the cardiologist. Hence, EGM data exported from CARTO system can be stored in a matrix of dimension $10 \times 2500$.

1) Data Labeling: Data labeling is performed by interventional cardiologists such that EGMs presenting spatiotemporal dispersion are annotated as "STD", also called gradient or substrate. We automatically merge remaining labels into the "non-STD" class. Moreover, a meticulous work is needed for standardizing the labels because the annotation nomenclature exported from CARTO system (encoded labels) differ from one patient to another. 


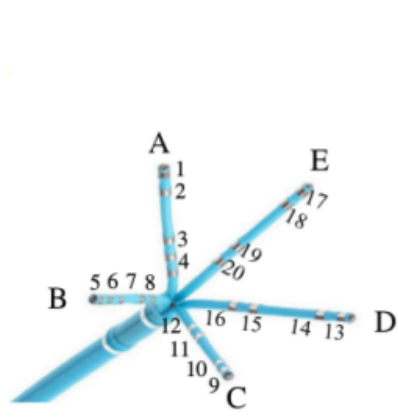

(A)
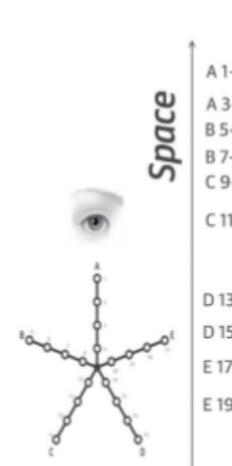
Winth or without fractionation)

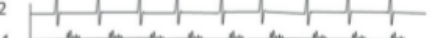

8 10. 11-12 No spatio-temporal Dispersion (With or without fractionation)

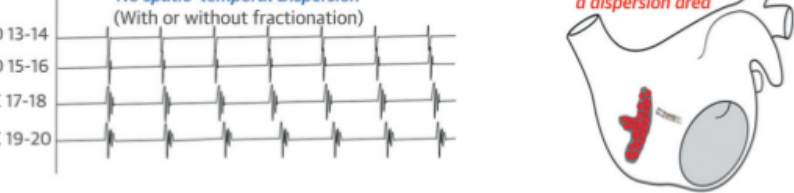

(B)

Fig. 1: (A) PentaRay multi-spline catheter (BioSense Webster Inc). (B) Dispersion areas defined and delineated via a mapping approach [6]. Channels A 1-2, A 3-4, B 5-6, B 7-8, C 9-10 and C 11-12 display STD contrarily to the remaining ones.

2) Circularity Transformation: Circularity transformation consists in replicating for each sample the first two channels (matrix rows) at the end of the sample. The new sample, originally with dimensions $10 \times 2500$, becomes $12 \times 2500$. This transformation allows us to mimic the circular arrangement of the PentaRay branches. Indeed, it captures the neighborhood information between bipoles of splines A and $\mathrm{E}$ of the catheter, as shown in Fig. 11.A).

\section{B. Data Representations}

Starting from the basic matrix format presented above, data can be reformatted in different ways to match different classification algorithms:

1) Matrix: The first classification scheme uses matrices of dimensions $12 \times 2500$, the result of applying the circularity transformation on raw recordings of the 10-lead EGMs. Matrices can also be treated as images with 2D CNN models like LeNet-STD, as proposed in [18] for a human activity recognition task.

2) 2D image: The image format consists in subplotting the curves of the 10 leads, one under the other, as it is currently done by CARTO system during the mapping phase.This 2D plot of EGMs is used in the hospital and represents the most easily understandable representation by the eye of an interventional cardiologist. We will check in the present study if this format is also convenient for ML classifiers.

3) 3D image: This format enables the use of CNN models conceived to train on 3D RGB images like in ImageNet [19] dataset (natural colored images). Hence, this data format enables the use of TL [7]. The 3D tensor is the result of stacking three $2 \mathrm{D}$ images along the depth dimension. The first slice (image) is a subplot of the ordered leads $\left\{\ell_{1}, \ell_{2}, \ell_{3}, \ell_{4}, \ell_{5}, \ell_{6}, \ell_{7}, \ell_{8}, \ell_{9}, \ell_{10}\right\}$, similarly to $2 \mathrm{D}$ image format. The second slice is the subplot of the leads shifted in a circular way. The leads order becomes $\left\{\ell_{2}, \ell_{3}, \ell_{4}, \ell_{5}, \ell_{6}, \ell_{7}, \ell_{8}, \ell_{9}, \ell_{10}, \ell_{1}\right\}$. The third slice represents a subplots of leads shifted twice as follows $\left\{\ell_{3}, \ell_{4}, \ell_{5}, \ell_{6}, \ell_{7}, \ell_{8}, \ell_{9}, \ell_{10}, \ell_{1}, \ell_{2}\right\}$. Similarly to the circularity transformation, the idea comes from the circularity of the PentaRay branches. The interest in shifting the leads remains in allowing the 3D filter to systematically analyze neighboring leads along the depth dimension as it already does along the spatial dimensions (matrix slice). For instance the first convolutional window captures leads $\left\{\ell_{1}, \ell_{2}, \ell_{3}\right\}$ across the three slices. This data format allows us to represent EGMs in a 3D tensor with a depth of three thus mimicking the shape of RGB images and allowing the use of algorithms requiring this input shape.

4) 1D signal: VAVp times series is a compact representation of multichannel recordings that has shown promising results in [11]. The VAVp distribution is also claimed to depend on STD pattern in [6]. As claimed in [6], VAVp time series is calculated as follows: 1) the VAV matrix contains in its rows the absolute values of each channel of the multilead EGM recording; 2) one-dimensional VAVp signal is computed as the maximal values at each time sample of VAV matrix over the leads (rows) dimension. The histograms of VAVp distribution $(h(\mathrm{VAV}))$ are shown in [6] to significantly depend on the presence of STD patterns. Numerical results showed that $h(\mathrm{VAVp})$ is peaky and concentrated around zero if the virtual PentaRay is positioned in non-STD areas but it gets more spread for EGMs recorded in STD areas of the heart as shown in Fig. 2. However, a recent study [11] showed that $h(\mathrm{VAVp})$ distribution is not a key feature in STD detection when analyzing the database used in the present study. However, the supervised classification of raw VAVp time series itself into STD vs. non-STD categories is promising. Results on the test set are good with values of accuracy, AUC, sensitivity and specificity around 90\% with low variability $\left(10^{-3}\right)$. Complementary metrics such as precision and F1 score are also computed and analyzed in the present work. Besides, a larger dataset of 35000 EGMs are included in the present paper compared to 23000 in [11].

\section{Classification Algorithms}

The ML algorithms trained to identify STD locations are the following:

1) SVM: SVM is widely used in biomedical data analysis like electrocardiogram classification [21]. The model maps the input feature space into a higher-dimensional space so 

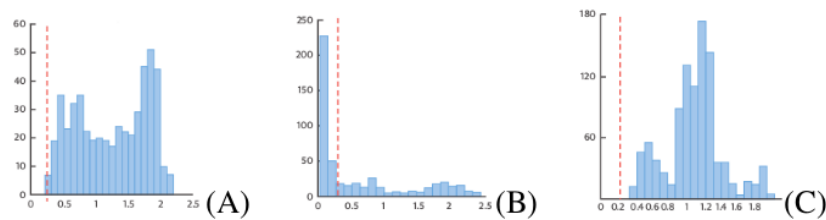

Fig. 2: VAVp distributions (histograms) obtained by positioning a virtual PentaRay in a human atrias with homogeneous substrate (A) at the center of the driver, reminiscent of patients' dispersion areas, and (B) at the periphery of the driver. (C) In the interstitial fibrosis condition [6].

that data samples from different classes become separable. Data is divided by a hyperplane in the original $n$-dimensional space, with a large gap [13]. SVM aims at maximizing the distance, called margin, between data points situated near the hyperplane, called support vectors. Maximizing the margin provides some confidence that new data points can be classified with more accuracy. Then, new samples are mapped into the new space and predicted to belong to a class or another based on the side of the hyperplane on which they fall. Looking for a suitable separable representation of data is called kernel trick.

2) PCA: PCA explores linear relationships between features and defines variables significance on the basis of their variance contribution. It projects data in a new space model formed by synthesized variables called principal components (PC). This is useful when large amounts of information may be approximated by a moderately complex model structure. Based on the estimation of the correlation structure of the variables, PCA evaluates the importance of a variable in a PC model through the size of its residual variance [12]. The choice to be examined is how many PC's $(r)$ adequately account for the total variation in the $n$-dimensional data sample $\mathbf{x}$. Among the existing criteria for choosing $r$, we opt for the cumulative percentage of total variation, also called hard threshold $(t)$. The optimal number of PC's is chosen as the smallest integer $r$ for which the cumulative percentage of total variation reaches or exceeds $t$. PCA allows us to reduce the dimensions of the input space by projecting the input data onto a space formed only by the first $r$ PCs

3) MLR: In statistics, logistic regression [2] estimates the probability of a given class or event by using the logistic function. MLR is identical to univariate logistic regression, but it considers more than one covariate. Let $p(\mathbf{x})$ be the model's estimation of the probability of an event that depends on $n$ covariates or independent variables. Then, inverting the logit formulation for modeling the probability gives:

$$
\left\{\begin{array}{l}
p: \mathbb{R}^{n} \rightarrow(0,1) \\
p(\mathbf{x})=\frac{e^{\beta_{0}+\beta_{1} x_{1}+\beta_{2} x_{2}+\cdots+\beta_{n} x_{n}}}{1+e^{\beta_{0}+\beta_{1} x_{1}+\beta_{2} x_{2}+\cdots+\beta_{n} x_{n}}} .
\end{array}\right.
$$

The estimates of the unknown parameters $\beta_{i}, i=$ $0,1,2, \ldots, n$, are derived through the maximization of a likelihood function. MLR is a baseline classifier in biomedical data analysis and electrograms classification [2]. Furthermore, MLR can be implemented as a fully connected (FC) neural network.

4) CNN: Artificial neural networks (NN) are commonly used in biomedical data classification [2], [22], [23]. A NN consists of a series of connected layers, each layer composed of a number of artificial neurons, called nodes, that have learnable weights. A CNN is a special NN composed of convolutional and pooling layers followed by FC ones. Each node of the convolutional (conv) layer receives some inputs, performs a dot product and optionally follows it with a nonlinearity. The output of each conv filter is called feature map. In pooling layers, the resolution of feature maps is reduced to increase the invariance of features to distortions.

(a) LeNet-STD architecture is inspired by the state of the art LeNet5, a baseline CNN that is both a good classifier and a computationally-affordable algorithm [14]. It has 60,000 parameters compared to AlexNet for instance that has 60 million parameters [7].

(b) VGG16 is a deep CNN designed by the Visual Geometry Group Lab, trained for ImageNet Large Scale Visual Recognition Challenge (ILSVRC) and achieving 92.7\% top5 test accuracy. ImageNet dataset contains 14 million images that belong to 1000 classes. VGG16 is composed of 5 conv blocks alternated by pooling layers and followed by FC ones. In total VGG16 has 16 layers with trainable weights [15]

5) $\boldsymbol{T L}:$ In terms of $\mathrm{ML}, \mathrm{TL}$ is a process where a NN $\left(M_{1}\right)$ is trained on a first problem then re-used in some way in a related problem. A new model is then formed by aggregating one or several layers from the trained model $M_{1}$ to additional layers, generally FC layers with trainable weights. The main benefits of TL consist in decreasing the training time for the new model and achieving a lower generalization error [7].

\section{Cross-Validation}

Cross-validation (CV) is a statistical tool commonly used in $\mathrm{ML}$ to quantify the generalization power of a classifier [24]. $k$-fold $\mathrm{CV}$ consists in: first, partitioning the entire datasets into $k$ subsets called folds; second, repeating model training $k$ times while considering, at each round, the $k^{\text {th }}$ fold as the test dataset and the remaining samples as the training dataset; finally, the estimation of the classifier's performance is given by averaging the validation results over the $k$ rounds. A rule of thumb is to choose $k$ equal to 5 or 10 [25]. Regarding the size of our dataset and the small number of STD samples (only 5\% of non-STD samples), we opt for 5-fold CV in order to keep an acceptable amount of STD test samples. In each CV round, the test dataset is partitioned into two equal-sized subsets that will form the new validation and test sets. This guarantees that the model does not see the test samples during the training phase.

\section{E. Performance Metrics}

A cost function is minimized during the learning phase of a classifier. In order to evaluate the model's classification performance, several metrics are assessed. The accuracy (Acc) metric is computed as the result of dividing the total number of correct predictions by the total number of predictions. However, accuracy is not enough to quantify 
classification performance when learning from imbalanced datasets. For this, it remains important to compute the confusion matrix whose elements are the true positives (TP), true negatives (TN), false positives (FP) and false negatives (FN). From these values, the following metrics are evaluated: 1) sensitivity, recall or true positive rate (TPR), measuring the proportion of actual STD samples that are correctly identified as such; 2) specificity or true negative rate (TNR), quantifying the proportion of actual non-STD samples that are correctly identified as such; 3) precision or positive predictive value (PPV), assessing how trustable the result is when the model answers that a point belongs to the STD class; 4) negative predictive value (NPV), or probability that a sample is actually non-STD when the model has classified it as such; and 5) $F_{1}$-score which stands for the harmonic mean of precision and sensitivity. The F1 score quantifies the balance between precision and recall values such that high recall accompanied with high precision values reflect that the STD class is perfectly handled by the model, low recall accompanied by high precision values reflect that the model cannot detect the STD class well but is sufficiently trustable when it does; high recall accompanied with low precision values reflect that the STD class is well detected but the model output for that class also includes non-STD samples, and low recall accompanied with low precision values reflect the STD class is poorly handled by the model. Then, the area under the Receiver Operating Characteristic (ROC) curve shortly named AUC is computed. The ROC curve is a graphical tool widely used to evaluate the performance of a binary classifier when varying the discrimination threshold [20].

\section{F. Data Augmentation}

One of the biggest and most frequent challenges encountered in deep learning remains the insufficient amount of data or the uneven class balance of samples within the training dataset. The high misrepresentation of STD samples in the multichannel EGM dataset leads to poor classification results in terms of sensitivity, precision and AUC. This data imbalance issue can be handled through adapted DA solutions. DA is a signal preprocessing tool that applies transformations to original samples of the minority STD class in order to synthesize new samples. It consists in forming a balanced super-dataset by replicating randomly STD samples until they reach the number of non-STD ones [9] [10]. A recent study benchmarked a set of DA solutions for the classification of the current STD multichannel EGM dataset [8]. The methods explored included random oversampling (ROS), undersampling, leads shift, time shift and time reversing. Each method has a different value of CIR (Tab. I in [8]). These methods were designed to preserve the integrity of STD pattern and were approved by partner interventional cardiologists. The classification performance of both a shallow CNN and MLR demonstrated that ROS is the best technique. Indeed, ROS increased the sensitivity value by $30 \%$ compared to learning from raw data while maintaining high values of specificity and AUC around $90 \%$. For this reason, we opt for ROS for the remaining data formats considered in the present study.

\section{Feature SElection FOR EGM Classification}

The association between the different data models and classifications algorithms is schematized in Fig. 3 A data model corresponds to the coupling between a data representation and a suitable classifier, as summarized next.

\section{A. Matrix Classification}

MLR and a shallow CNN, called LeNet-STD, are used for the classification of EGM matrices. LeNet-STD is chosen as a proof of concept that 2D CNN architectures are adapted to STD detection. We study the effect of the receptive field of the network's first conv layer, denoted $f_{\text {size }}$. We recall that the STD pattern can be detected on a minimum of three bipoles positioned on two adjacent PentaRay branches and the average AFCL value is typically $200 \mathrm{~ms}$. Hence, a natural choice is $f_{\text {size }}=4 \times\left(\alpha_{1} \cdot\right.$ AFCL $)$, with $\alpha_{1} \in \mathbb{Q}, \alpha_{1} \geq 1$. The value $\alpha_{1}=\frac{3}{2}$ is proposed in [8], thus capturing cardiac activations along a minimum of one and a half AFCL, and yielding $f_{\text {size }}=4 \times 300$. The corresponding model is denoted LeNet-STD $(4 \times 300)$. We investigate further settings such as $f_{\text {size }}=4 \times 3$ and $f_{\text {size }}=4 \times 200$, whose models are denoted LeNet-STD $(4 \times 3)$ and LeNet-STD $(4 \times 200)$, respectively. An $f_{\text {size }}$ of $4 \times 200$ is conceived to capture exactly an entire AFCL.

\section{B. 2D Image Classification}

A classical window size for image classification is $3 \times 3$ [15]. Indeed, $f_{\text {size }}=3 \times 3$ is the smallest receptive field to capture the patterns of left/right, up/down and center, as explained in [15]. Hence, MLR and LeNet-STD $(3 \times 3)$ are used for EGM-plot image classification.

\section{3D Image Classification}

We perform the classification of 3D images with a deep CNN called VGG16-EGM. VGG16-EGM is the result of transferring the VGG16 architecture. Indeed, we re-use the first trained conv blocks of VGG16 model. The transferred layers serve as feature extractor. Their output is fed to three consecutive FC layers with 1024, 128 and 2 nodes, respectively. Only FC layers have learnable weights. Hence, VGG16-EGM is adapted to 3D image classification and can be trained on the EGM dataset.

\section{VAVp Classification}

In addition to MLR, SVM is another baseline linear classifier in ML. However, classifying VAVp time series was not possible with SVM because this input has 2500 covariates and SVM is not adapted to such a large amount of variables. We propose to reduce the dimensions of VAVp signals with PCA, as explained in Sec. III-C.2 We use the hard threshold technique at $95 \%$ of explained variance to decide the number $r$ of PCs to keep. Then, we feed the reduced version of VAVp to SVM. Linear and Gaussian kernels are benchmarked. The models are denoted respectively PCA-

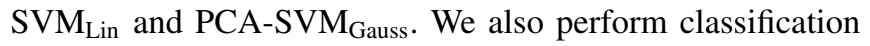
with a shallow 1D CNN (CNN-1D). 


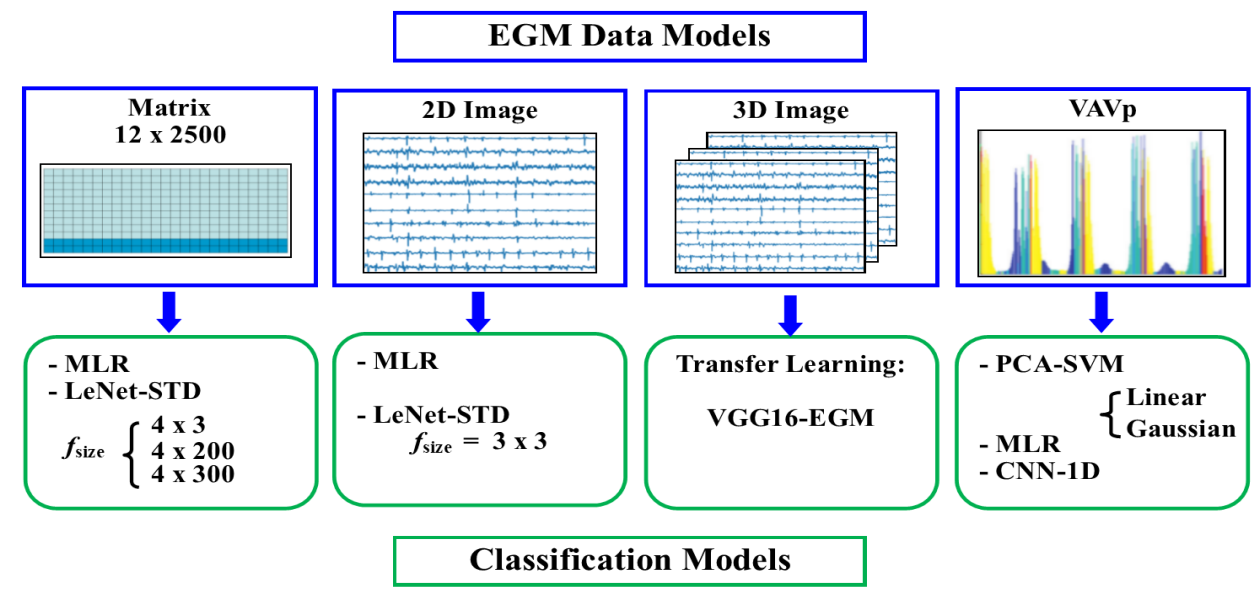

Fig. 3: Classification models adapted to the different EGM data features.

Implementation: ML experiments are conducted within the Docker environment, running on Quadro P6000 GPU and Ubuntu 19.10 operating system using Python 3.6.9 programming language, Tensorflow 2.1.0 deep learning library for GPU and Keras API.

\section{RESULTS}

\section{A. Study Dataset}

CARTO data of 15 patients are exported in the present study from the database of the Cardiology Department of Nice University Hospital Center (CHU Pasteur), whose Ethics Committee approved the proposed research. Cartographies of both right and left arias are merged. The study dataset includes a cohort of 35563 10-channel EGM signals of length $2.5 \mathrm{~s}$ acquired from both right (RA) and left atria (LA). The recordings include 1804 STD and 33759 nonSTD samples. The baseline information of the study patients is given in Tab. I The population is aged 64 years on average and is composed of $80 \%$ male and $20 \%$ female patients. The average initial AF cycle in left atrial appendage (LAA) is $156 \mathrm{~ms}$. On average, the ablation intervention lasts $2 \mathrm{~h} 36 \mathrm{~min}$ and requires $155 \mathrm{RF}$ shots for a total of $53 \mathrm{~min} 57 \mathrm{~s}$. The class imbalance ratio (CIR) of our dataset is given by:

$$
\mathrm{CIR}=\frac{\# \mathrm{STD}}{\# \text { non-STD }} \approx 5 \%
$$

where \# refers to the number of samples. Based on our finding in the comparative study [8] and due to the extremely low value of CIR, we opt for ROS to handle the lack of a sufficient amount of STD samples, as justified in Sec. III-F.

\begin{tabular}{|c|c|}
\hline Feature & Value (mean \pm std) \\
\hline Gender & $12 \mathrm{Male}+3 \mathrm{Female}$ \\
\hline Age (year) & $64 \pm 12$ \\
\hline Initial AF cycle in LAA (ms) & $156 \pm 12$ \\
\hline Procedure duration & $2 \mathrm{~h} 36 \mathrm{~min} \pm 42 \mathrm{~min}$ \\
\hline RF duration & $53 \mathrm{~min} 57 \mathrm{~s} \pm 12 \mathrm{~min} 15 \mathrm{~s}$ \\
\hline Number of RF shots & $155 \pm 61$ \\
\hline
\end{tabular}

TABLE I: Baseline information about patient's population.

\section{B. Classification Results}

The identification of STD locations is performed with several ML techniques including TL. For this purpose, we benchmark the different EGM classification models detailed in Sec. IV] Their performance on test sets are given in Tab. II. where values are calculated as the average over 5 -fold $\mathrm{CV}$, as explained in Sec. III-D All standard deviations are inferior to $10^{-2}$. Hence, all trained models have a low generalization error.

1) Matrix Classification: Both LeNet-STD with its three settings of $f_{\text {size }}$ and MLR yield good and comparable performance. However, LeNet-STD slightly overperforms MLR, as the latter obtains values of AUC and F1 equal to 92\% and 53\% respectively. However, The average values of AUC and F1 are $94 \%$ and $58 \%$ for LeNet-STD. The choice of $f_{\text {size }}$ in LeNet-STD has a small impact on the classification performance but has an important effect on the computational cost. Indeed, F1 scores are 58\%, 59\% and $60 \%$, respectively, with LeNet-STD $(4 \times 3)$, LeNet-STD $_{(4 \times 200)}$ and LeNet-STD $(4 \times 300)$ respectively. However, the training times of LeNet-STD $(4 \times 300)$ and LeNet-STD $(4 \times 200)$ are very high compared to LeNet-STD $(4 \times 3)$ as shown in Tab. III.

2) 2D Image Classification: Second, we check if classifying $2 \mathrm{D}$ images would give good performance because this is the most intuitive input format. In practice, interventional cardiologist analyze visually EGM subplots to detect STD pattern, so this format seems particularly suitable in this context. However, results in Tab. III show no significant enhancement in performance with 2D images compared to EGM matrices. Here again, we notice that LeNet-STD yields a better performance, with an F1 score of nearly $60 \%$, comparing favorably to the $52 \%$ provided by MLR.

3) 3D Image Classification: Applying TL of VGG16 model to 3D images is performed as a proof of concept. VGG16-EGM yields good classification performance with values of Acc, AUC and F1 of $93 \%, 93 \%$ and $55 \%$, respectively. This shows that extracting features from 3D plots with the use of conv blocks, trained on a different application (natural images), does work. However, besides the 
heavy computational cost of VGG16-EGM, as highlighted in Tab III] synthesizing 3D images reveals heavy too. Several extensions were benchmarked for storing and reading images in an optimal way.

4) VAVp Classification: The overall classification performance of VAVp time series is also good but less effective than the remaining data representations. For instance, the values of $\mathrm{F} 1$ score do not exceed $50 \%$. Tab $\amalg$ shows that the performance of CNN-1D is slightly better than that of MLR. MLR beats significantly PCA-SVM, mainly in terms of F1 score. Moreover, the Gaussian kernel outperforms the linear one. The VAVp format does not yield the best value in any of the performance metrics considered in this evaluation and results in the lowest F1 scores, all below $46 \%$.

\section{Discussion}

Automatic classification of STD EGMs is possible using suitable ML techniques. Based on the results reported in this work, we can claim that classifying raw EGMs stored in matrices with LeNet-STD $(4 \times 3)$ yields a good balance between performance and computational cost. If we observe the values of precision and F1 across all experiments (Tab. II), we notice poor values $(30 \%$ with VAVp and inferior to $60 \%$ with the remaining features) even though values of TPR are high around $80 \%$ and $90 \%$ (except with PCASVM). However, values of PPV and F1 are high for the training set that is balanced with ROS. This low precision on the test sets can be explained by the fact that both test and validation sets remain highly imbalanced. Hence, even though the trained models can identify better STD samples when the training set is augmented, as highlighted in [8], information about STD class is still less rich than that of nonSTD due the redundancy introduced by ROS. A low precision also indicates that the number of FP is important compared to that of TP. This can be acceptable in biomedical data analysis. On the one hand, TPR rate is high which means that true STDs are well detected. On the other hand, it is always preferable to detect non-STDs as STDs than conversely. This allows the model to tag these FP predictions during the mapping phase and give the cardiologist the opportunity to analyze them later, before ablation. Also, Tab. III shows that our solution can be deployed in real time since the prediction time (inference) of a new data sample can be performed in less than a second (maximal prediction time equal to $0.944 \mathrm{~s}$ for VGG16-EGM). If more efficient computational resources are deployed, prediction time can be further decreased. The overall performance achieved with our proposed solutions is good with values of accuracy and AUC around 90\%, but better results are expected in a medical decision-aid tool. An accuracy of $95 \%$ should be attained for the solution to be considered as sufficiently reliable for clinical use. Also, a higher number of patients would provide a richer EGM database with increased variability across patients.

\section{Conclusions and Perspectives}

Automatic detection of atrial areas with STD pattern is a valuable decision-aid tool that would help interventional cardiologists identify potential ablation sites for treating persistent $\mathrm{AF}$ in a faster and more reliable manner than the current visual inspection. To identify STD, several features are extracted from EGM recordings either automatically with TL and end-to-end NN training or in a hand-crafted fashion with VAVp time series. These different data representations are classified with the use of adapted ML algorithms giving rise to a variety of EGM classification models. Moreover, we study the effect of some hyperparameters and settings like the choice of receptive fields and kernels in SVM. Based on the analysis of both the classification performance on the test set and the computational cost of the different classification models, the best performance is achieved with LeNet-STD and $f_{\text {size }}=4 \times 3$ for classifying raw EGM matrices. The average performance over 5-fold CV reaches $94 \%$ of accuracy and AUC added to an $\mathrm{F} 1$-score of $60 \%$. The low precision and F1-score can be explained by the insufficient amount of STD samples even thought the class imbalance issue is alleviated with DA during the training phase. On the other hand, VGG16-EGM demonstrates that extracting features with conv blocks of the VGG16 model, trained on natural images, works as well as shallower architectures like LeNetSTD, but is very expensive computationally. By providing interventional cardiologists with a real-time objective measure of STD, the proposed solution presents the potential to improve the efficiency and effectiveness of this fully patienttailored catheter ablation approach for treating persistent AF. Future work should investigate alternative ML models like long short-term memory (LSTM) neural networks, as they are well adapted to time series classification [26].

\section{REFERENCES}

[1] Q. Yao, R. Wang, X. Fan, J. Liu and Y. Li, "Multi-class arrhythmia detection from 12-lead varied-length ECG using attention-based timeincremental convolutional neural network," Information Fusion, 53, pp. 174-182, 2020.

[2] S. Dreiseitl and O. M. Lucila, "Logistic regression and artificial neural network classification models: a methodology review," Journal of Biomedical Informatics, 35(5-6), pp. 352-359, 2002.

[3] V. Zarzoso, D.G. Latcu, A.R. Hidalgo-Muñoz, M. Meo, O. Meste, I. Popescu and N. Saoudi, "Non-invasive prediction of catheter ablation outcome in persistent atrial fibrillation by fibrillatory wave amplitude computation in multiple electrocardiogram leads," Archives of Cardiovascular Diseases, 109(12), pp. 679-688, 2016.

[4] C. T. January, L. S. Wann, H. Calkins, L. Y. Chen, J. E. Cigarroa, J. C. Cleveland, et al. "2019 AHA/ACC/HRS focused update of the 2014 AHA/ACC/HRS guideline for the management of patients with atrial fibrillation: a report of the American College of Cardiology/American Heart Association Task Force on Clinical Practice Guidelines and the Heart Rhythm Society," Journal of the American College of Cardiology, 74(1), pp. 104-132, 2019.

[5] A. Verma, C.Y. Jiang, T.R. Betts and et al., "Approaches to catheter ablation for persistent atrial fibrillation," New England Journal of Medicine, 372(19), pp. 1812-1822, 2015.

[6] J. Seitz, C. Bars, G. Théodore and et al., "AF ablation guided by spatiotemporal electrogram dispersion without pulmonary vein isolation: a wholly patient-tailored approach," Journal of the American College of Cardiology, 69(3), pp. 303-321, 2017.

[7] I. Goodfellow, Y. Bengio and A. Courville, Deep Learning, MIT Press, 2016. www.deeplearningbook.org.

[8] A. Ghrissi, D. Almonfrey, R. Almeida, F. Squara, V. Zarzoso and J. Montagnat, "Data augmentation for automatic identification of spatiotemporal dispersion electrograms in atrial fibrillation ablation using machine learning," in: Proc. 42nd Annual International Conference 


\begin{tabular}{|c|c|c|c|c|c|c|c|c|}
\hline \multirow{2}{*}{ Feature } & \multirow{2}{*}{ Model } & \multicolumn{7}{|c|}{ Test classification performance } \\
\cline { 3 - 9 } & & Acc & TPR & TNR & AUC & PPV & NPV & F1 \\
\hline \multirow{3}{*}{ Matrix } & MLR & 0.924 & 0.902 & 0.847 & 0.929 & 0.389 & 0.991 & 0.533 \\
\cline { 2 - 9 } & LeNet-STD $_{(4 \times 3)}$ & 0.936 & 0.911 & 0.862 & 0.94 & 0.436 & 0.992 & 0.579 \\
\cline { 2 - 9 } & LeNet-STD $_{(4 \times 200)}$ & 0.937 & $\mathbf{0 . 9 2 9}$ & 0.881 & 0.940 & 0.443 & $\mathbf{0 . 9 9 3}$ & 0.589 \\
\cline { 2 - 9 } & LeNet-STD $_{(4 \times 300)}$ & $\mathbf{0 . 9 4 0}$ & 0.928 & 0.879 & $\mathbf{0 . 9 4 3}$ & $\mathbf{0 . 4 5 3}$ & 0.99 & $\mathbf{0 . 5 9 8}$ \\
\hline \multirow{3}{*}{ 2D Image } & MLR & 0.873 & 0.913 & 0.852 & 0.874 & 0.398 & 0.991 & 0.517 \\
\cline { 2 - 9 } & LeNet-STD $_{(3 \times 3)}$ & 0.939 & 0.911 & 0.88 & $\mathbf{0 . 9 4 3}$ & 0.452 & $\mathbf{0 . 9 9 3}$ & 0.597 \\
\hline \multirow{3}{*}{ 3D Image } & VGG16-EGM $_{\text {VAVp }}$ & 0.926 & 0.88 & $\mathbf{0 . 9 2 8}$ & 0.925 & 0.406 & 0.992 & 0.553 \\
\cline { 2 - 9 } & PCA-SVM $_{\text {Lin }}$ & 0.791 & 0.670 & 0.535 & 0.805 & 0.128 & 0.970 & 0.207 \\
\cline { 2 - 9 } & PCA-SVM $_{\text {Gaus }}$ & 0.837 & 0.732 & 0.616 & 0.849 & 0.179 & 0.976 & 0.277 \\
\cline { 2 - 9 } & MLR & 0.888 & 0.891 & 0.807 & 0.892 & 0.286 & 0.988 & 0.423 \\
\cline { 2 - 9 } & CNN-1D & 0.902 & 0.890 & 0.817 & 0.907 & 0.321 & 0.989 & 0.460 \\
\hline
\end{tabular}

TABLE II: Average classification performance on test set through 5-fold CV.

\begin{tabular}{|c|c|c|c|c|}
\hline \multirow{2}{*}{ Feature } & \multirow{2}{*}{ Model } & \multicolumn{2}{|c|}{ Training cost } & \multirow{2}{*}{ Prediction time (s) } \\
\cline { 3 - 5 } & & time & nb $_{\text {tr-par }}$ & \\
\hline \multirow{3}{*}{ Matrix } & MLR & $00: 01: 49$ & 400,672 & 0.139 \\
\cline { 2 - 5 } & LeNet-STD $_{(4 \times 3)}$ & $00: 11: 04$ & $1,289,458$ & 0.643 \\
\cline { 2 - 5 } & LeNet-STD $_{(4 \times 200)}$ & $00: 24: 57$ & $1,214,322$ & 0.625 \\
\cline { 2 - 5 } & LeNet-STD $_{(4 \times 300)}$ & $00: 37: 51$ & $1,175,922$ & 0.616 \\
\hline \multirow{2}{*}{ 2D Image } & MLR & $01: 08: 57$ & 400,672 & 0.144 \\
\cline { 2 - 5 } & LeNet-STD $_{(3 \times 3)}$ & $01: 10: 15$ & $1,289,362$ & 0.618 \\
\hline \multirow{2}{*}{ 3D Image } & VGG16-EGM & $14: 35: 28$ & $100,795,778$ & 0.944 \\
\hline \multirow{2}{*}{ VAVp } & PCA-SVM $_{\text {Lin }}$ & $00: 10: 14$ & $O\left(m^{2} n\right)$ & 0.003 \\
\cline { 2 - 5 } & PCA-SVM $_{\text {Gaus }}$ & $00: 55: 32$ & $O\left(m^{3} r\right)$ & 0.002 \\
\cline { 2 - 5 } & MLR & $00: 12: 39$ & 400,672 & 0.141 \\
\cline { 2 - 5 } & CNN-1D & $00: 11: 25$ & 80,066 & 0.585 \\
\hline
\end{tabular}

TABLE III: Computational cost of training on balanced dataset and predicting the label of a test data sample. Time is given as hours(hh):minutes(mm):seconds(ss). Symbol $\mathrm{nb}_{\text {tr-par }}$ stands for the number trainable parameters, which might be inferior to the total number of parameters in case of TL when used as a feature extractor. The number of variables is $n=2500$ and $r \approx 300$.

of the IEEE Engineering in Medicine and Biology Society, Montreal, Canada, July 2020.

[9] M. Agnieszka and M. Grochowski, "Data augmentation for improving deep learning in image classification problem," in: Proc. IEEE International Interdisciplinary PhD Workshop, May 2018.

[10] C. Shorten and L. K. Taghi, "A survey on image data augmentation for deep learning," Journal of Big Data, 6(1), p. 60, 2019.

[11] A. Ghrissi, F. Squara, V. Zarzoso and J. Montagnat, "Identification of spatiotemporal dispersion electrograms in persistent atrial fibrillation ablation using maximal voltage absolute values," in: Proc. 28th European Signal Processing Conference, Amsterdam, The Netherlands, Jan. 2021.

[12] I. T. Jolliffe and C. Jorge, "Principal component analysis: a review and recent developments," Philosophical Transactions of the Royal Society A: Mathematical, Physical and Engineering Sciences, 374(2065), pp. 2015-0202, 2016.

[13] B. E Boser, M. G. Isabelle and N. V. Vladimir, "A training algorithm for optimal margin classifiers," in Proc. $5^{\text {th }}$ Annual Workshop on Computational Learning Theory, USA, pp. 144-152, 1992.

[14] Y. LeCun, L. Bottou, Y. Bengio and et al., "Gradient-based learning applied to document recognition," Proceedings of the IEEE, 86(11), pp. 2278-2324, 1998.

[15] K. Simonyan and A. Zisserman, "Very deep convolutional networks for large-scale image recognition," in: Proc. International Conference on Learning Representations, San Diego, CA, USA, May 2015.

[16] J. Malmivuo and R. Plonsey, Bioelectromagnetism: Principles and Applications of Bioelectric and Biomagnetic Fields, Oxford University Press, NY, USA, 1995.

[17] K. Nademanee, J. McKenzie, E. Kosarand et al., "A new approach for catheter ablation of atrial fibrillation: mapping of the electrophysiologic substrate," Journal of the American College of Cardiology, 43(11), pp. 2044-2053, 2004.

[18] J. Yang, M.N. Nguyen, P.P. San, X. Li and S. Krishnaswamy, "Deep convolutional neural networks on multichannel time series for human activity recognition," in: Proc. 24th International Joint Conference on Artificial Intelligence, Buenos Aires, Argentina, July 2015.

[19] J. Deng, W. Dong, R. Socher and et al., "ImageNet: A large-scale hierarchical image database," in: Proc. IEEE Conference on Computer Vision and Pattern Recognition, Miami, FL, USA, June 2009, pp. 248255.

[20] K. Hajian-Tilaki, "Receiver operating characteristic (ROC) curve analysis for medical diagnostic test evaluation," Caspian Journal of Internal Medicine, 4(2), p. 627, 2013.

[21] F. Melgani and B. Yakoub, "Classification of electrocardiogram signals with support vector machines and particle swarm optimization," IEEE Transactions on Information Technology in Biomedicine, 12(5), pp. 667-677, 2008.

[22] B. Pyakillya, N. Kazachenko and N. Mikhailovsky. "Deep learning for ECG classification," Journal of Physics: Conference Series, 913(1), IOP Publishing, 2017.

[23] A.Y. Hannun, P. Rajpurkar, M. Haghpanahi, and et al., "Cardiologistlevel arrhythmia detection and classification in ambulatory electrocardiograms using a deep neural network," Nature Medicine, 25(1), p. $65,2019$.

[24] R. Kohavi, "A study of cross-validation and bootstrap for accuracy estimation and model selection," in: Proc. 14th International Joint Conference on Artificial Intelligence, Montreal, Canada, Aug. 1995.

[25] M. Kuhn and J. Kjell, Applied Predictive Modeling, Springer, 26, p. 70, 2013.

[26] A.Y. Hannun, P. Rajpurkar, M. Haghpanahi and et al. "Cardiologistlevel arrhythmia detection and classification in ambulatory electrocardiograms using a deep neural network," Nature Medicine, 25, pp. 6569, 2019. 